\title{
High intensity training induces alteration of the ubiquitin-proteasome system gene expression profile and structural changes in the ovaries
}

\author{
RUOHONG XIONG ${ }^{1}$, XUE REN $^{1}$, GUIMING WANG ${ }^{2}$, GUOQING CHEN $^{2}$, \\ HUIJUN YANG ${ }^{2}$, QIONGJIA YUAN ${ }^{1}$ and HONGYING ZHOU ${ }^{2}$ \\ ${ }^{1}$ Department of Sports Medicine, Chengdu Sports University; ${ }^{2}$ Department of Anatomy, School of Preclinical \\ and Forensic Medicine, Sichuan University, Chengdu, Sichuan, P.R. China
}

Received November 22, 2011; Accepted February 15, 2012

DOI: $10.3892 / \mathrm{mmr} .2012 .807$

\begin{abstract}
The female reproductive system is highly sensitive to physiological stress. However, the mechanism(s) involved in dysfunction of the ovaries induced by high intensity exercise training remain unknown. In the present study, we established a rat model of high intensity exercise training, and investigated the morphological and functional changes in ovaries. Furthermore, we profiled the differential gene expression in the ovaries between high intensity exercise training rats and control rats by restriction fragment differential display PCR. Differentially expressed genes were validated by real-time PCR. The results showed that the weight and microstructure of the ovaries were significantly altered in the exercise training group rats. In addition, the levels of estradiol, progesterone, luteinizing hormone and follicle-stimulating hormone were significantly decreased in the exercise training group rats compared with the control group rats. Immunohistochemistry revealed weak expression of estradiol, estrogen receptor and progesterone receptor in ovarian tissues from the high intensity exercise training group compared with the control group. Furthermore, the gene expression profiles of the exercise training and control group rats were analyzed, and differentially expressed genes, such as those involved in the ubiquitin-proteasome system, were identified. Our results indicate that high intensity exercise training can suppress the cellular function of the ovary glands. The mechanism may involve a series of alterations in the expression of genes particularly associated with the ubiquitin-proteasome system.
\end{abstract}

Correspondence to: Dr Hongying Zhou, Department of Anatomy, School of Preclinical and Forensic Medicine, Sichuan University, Sichuan, P.R. China

E-mail: eaglezhyxzy@163.com

Key words: ubiquitin-proteasome system, high intensity training, ovary

\section{Introduction}

Female athletes have become increasingly physically active over the past several decades. Previous studies indicate that physical activity is associated with numerous benefits in terms of health, including a lower incidence of cardiovascular disease, non-insulin-dependent diabetes mellitus, osteoporotic fractures, colon cancer and perhaps breast cancer (1). Regular physical activity also helps to maintain a healthy body weight, thus reducing the incidence of diseases related to obesity (2). While exercise provides substantial health benefits, rigorous physical activity is also associated with a series of risks for female athletes. The female reproductive system is highly sensitive to physiological stress. Delayed menarche, amenorrhea or oligomenorrhea occur in 6-79\% of female athletes (3). It was confirmed that high levels of physical stress due to a long period of high intensity exercise training may induce a lower level of progesterone in the midluteal phase and a shorter luteal phase, even infertility (4). Yet, the mechanisms involved in the dysfunction of ovaries induced by high intensity exercise training remain unknown.

The ubiquitin-proteasome system is the major system involved in protein degradation and consists of three classes of ubiquitin enzymes: ubiquitin-activating enzyme (E1), ubiquitin-conjugating enzyme (E2) and ubiquitin protein ligase (E3) (5). It has been confirmed that the ubiquitin-proteasome system is involved in numerous cellular processes such as cell cycle progression, cell proliferation and apoptosis and gene transcription regulation (6). Recently, studies have demonstrated that the ubiquitin proteasome system impairs ovarian development and oocyte meiosis (7-10). It has also been confirmed that exercise training affects the expression of genes in the ubiquitin-proteasome system in various tissues such as skeletal muscle and myocardium and nerve tissue in the human body or in animal models, resulting in protein degradation and cell apoptosis (11-13). However, the influence of high intensity exercise training on the gene expression profile of the ubiquitin-proteasome system in the ovaries is unknown.

Therefore, in the present study, we established a rat model of high intensity exercise training, and the restriction fragment differential display-polymerase chain reaction (RFDD-PCR) 
was employed to investigate the gene expression profiles involved in the dysfunction of ovaries induced by high intensity training.

\section{Materials and methods}

Animals and the high intensity training program. Twenty healthy non-copulating female Sprague-Dawley rats, body mass $220 \pm 3 \mathrm{~g}$, were selected and randomly divided into a high intensity training group $(n=10)$ and a control group $(n=10)$. All rats were housed under a 12-h light/dark cycle with free access to food and water. The exercise training protocol consisted of swimming for 8 weeks, and the rats assigned to the exercise training group trained 6 days/week and twice daily as previously described (14). Briefly, after 1 week of familiarizing the rats to the swimming apparatus, the training period continued for $15 \mathrm{~min}$ during the first week, with a gradual increase in training time to $120 \mathrm{~min}$ in week 4; this schedule was subsequently maintained until the termination of the experiment. From week 4 to the experiment end, the animals were loaded with $0.5 \%$ of body weight, which was gradually increased to $2 \%$ of body weight in week 8 . The excercise training rats and control rats were sacrificed $48 \mathrm{~h}$ after the last training session to examine the effects of total training but not the effects resulting from the last training. This study was approved by the Institutional Animal Ethics Committee of Chengdu Sports University.

Measurement of bone mineral density and plasma hormones. Before and after the exercise training, the bone mineral density (BMD) $\left(\mathrm{g} / \mathrm{cm}^{2}\right)$ of all animals was measured using a dual energy X-ray absorptiometer (DXA) (Lunar Radiation, Madison, WI, USA). Following the DAX scan, peripheral blood of all animals was prepared and levels of reproductive hormones, such as estradiol, progesterone, luteinizing hormone ( $\mathrm{LH})$, and follicle-stimulating hormone (FSH), were determined by ELISA in accordance with the user's manual.

Tissue preparation and immunohistochemistry. The animals were sacrificed with a high dose of sodium thiopentone (100 mg/kg body weight). The ovary and uterus were carefully dissected and the weight was measured. Left ovary samples for each rat were placed in $4 \%$ paraformaldehyde and $0.1 \mathrm{~mol} / \mathrm{l}$ phosphate-buffered saline for $24 \mathrm{~h}$ at $4^{\circ} \mathrm{C}$. Right ovary samples were frozen in liquid nitrogen and stored at $-80^{\circ} \mathrm{C}$ until further analysis. Paraffin sections $(5-\mu \mathrm{m})$ were mounted on poly-L-lysine-coated (Sigma-Aldrich) slides and stained with hematoxylin and eosin (H\&E) (Sigma-Aldrich). For the immunohistochemical staining, the sections were deparaffinized in xylene and immersed in graded ethanol and distilled water. Immunohistochemical staining was performed using the avidin-biotin peroxidase complex (ABC) method, according to the manufacturer's instructions. The primary antibody for estradiol (1:500, Santa Cruz Biotechnology, Inc.), estrogen receptor (ER) (1:250, Santa Cruz Biotechnology, Inc.) and progesterone receptor (PR) (1:250, Santa Cruz Biotechnology, Inc.) were used. The primary antibody was omitted as a negative control for the immunostaining.

Restriction fragment differential display-polymerase chain reaction ( $R F D D-P C R)$. Total-RNA of samples from the
Table I. Sequence of the primers used for real-time PCR.

\begin{tabular}{|c|c|c|}
\hline $\begin{array}{l}\text { Gene } \\
\text { name }\end{array}$ & Primer sequence $\left(5^{\prime}->-3^{\prime}\right)$ & $\begin{array}{l}\text { Product } \\
\text { length (bp) }\end{array}$ \\
\hline Psmb5 & $\begin{array}{l}\text { CCTACATTGCTTCCCAGACAGTG } \\
\text { GACCGAGATGCGTTCCTTGTT }\end{array}$ & 158 \\
\hline Psmdl & $\begin{array}{l}\text { GTGGTCTCTTTGTTGTCCGAGAG } \\
\text { CGTAGTTCACAGGGTCATTGGTC }\end{array}$ & 142 \\
\hline Psmd8 & $\begin{array}{l}\text { ACAAGGTGTTCCTGGCTAAGG } \\
\text { TTGGGTGTGCTGAAGAAGAGG }\end{array}$ & 166 \\
\hline Ube3a & $\begin{array}{l}\text { GAGTAGATGAGGGAGGCGTTTC } \\
\text { ACCCAGGACTATGCCAATCAGAG }\end{array}$ & 170 \\
\hline Usp10 & $\begin{array}{l}\text { GACGCCTATCACTGGCATTTTC } \\
\text { CCTGGACTGTGCGAATCTTGT }\end{array}$ & 137 \\
\hline$U s p 9 x$ & $\begin{array}{l}\text { CCCTCTTTAGCAGTTGTCCTGTG } \\
\text { GCCAGTGTATGGTCTTCTTTCG }\end{array}$ & 122 \\
\hline$\beta$-actin & $\begin{array}{l}\text { ACGGTCAGGTCATCACTATCG } \\
\text { GGCATAGAGGTCTTTACGGATG }\end{array}$ & 155 \\
\hline
\end{tabular}

Table II. Body weight and weights of the ovary and uterus and levels of plasma hormones in the two groups.

\begin{tabular}{lcc}
\hline & Control rats & $\begin{array}{c}\text { Exercise } \\
\text { training rats }\end{array}$ \\
\hline Body weight $(\mathrm{g})$ & $256.06 \pm 21.77$ & $228.2 \pm 15.94$ \\
Ovary weight $(\%$ body weight) & $5.07 \pm 1.05$ & $3.5 \pm 1.27$ \\
Uterus weight (\% body weight) & $14.3 \pm 3.77$ & $10.91 \pm 2.59$ \\
Estradiol $(\mathrm{ng} / \mathrm{ml})$ & $1.56 \pm 0.48$ & $1.27 \pm 0.34$ \\
Progesterone $(\mathrm{pg} / \mathrm{ml})$ & $73.81 \pm 23.42$ & $45.70 \pm 18.44$ \\
FSH $(\mathrm{mIU} / \mathrm{ml})$ & $8.57 \pm 1.22$ & $7.34 \pm 0.99$ \\
LH $(\mathrm{mIU} / \mathrm{ml})$ & $2.13 \pm 0.30$ & $1.32 \pm 0.24$ \\
\hline
\end{tabular}

FSH, follicle-stimulating hormone; LH, luteinizing hormone.

ovaries of the two groups was extracted respectively by TRIzol (Invitrogen) according to the manufacturer's instructions. RFDD-PCR for each sample was accomplished by DisplayPROFILE ${ }^{\mathrm{TM}}$ kits (cat. nos. 600-100, 615-100, 616-100, 617-100, 618-100) provided by Qbiogene, Inc. and MP Biomedicals, Inc. (Carlsbad, CA, USA) as previously described and used in our laboratory $(15,16)$. After RFDD-PCR, $15 \mu 1$ loading buffer was added, and the samples were heated to $85^{\circ} \mathrm{C}$ for 5 min and placed directly on ice. Each sample $(5 \mu \mathrm{l})$ was loaded on a $7 \%$ urea polyacrylamide gel that had been pre-run $30 \mathrm{~min}$ at $60 \mathrm{~W}$ in a High-Voltage Vertical Electrophoresis system (Bio-Rad, Hercules, CA, USA), and 0.6X Tris-borate (TBE)-ethylenediaminetetraacetic acid (EDTA) was used as electrophoresis buffer. Gels were scanned with Typhoon 9200 Variable Mode Imager (GE Healthcare). The scanning parameters consisted of wavelength of 33/670 nm, PMT voltage of $625 \mathrm{~V}$ and scanning precision of $200 \mathrm{~lm}$. On the basis of the linear correlation between the molecule weight (base pairs, bp) 

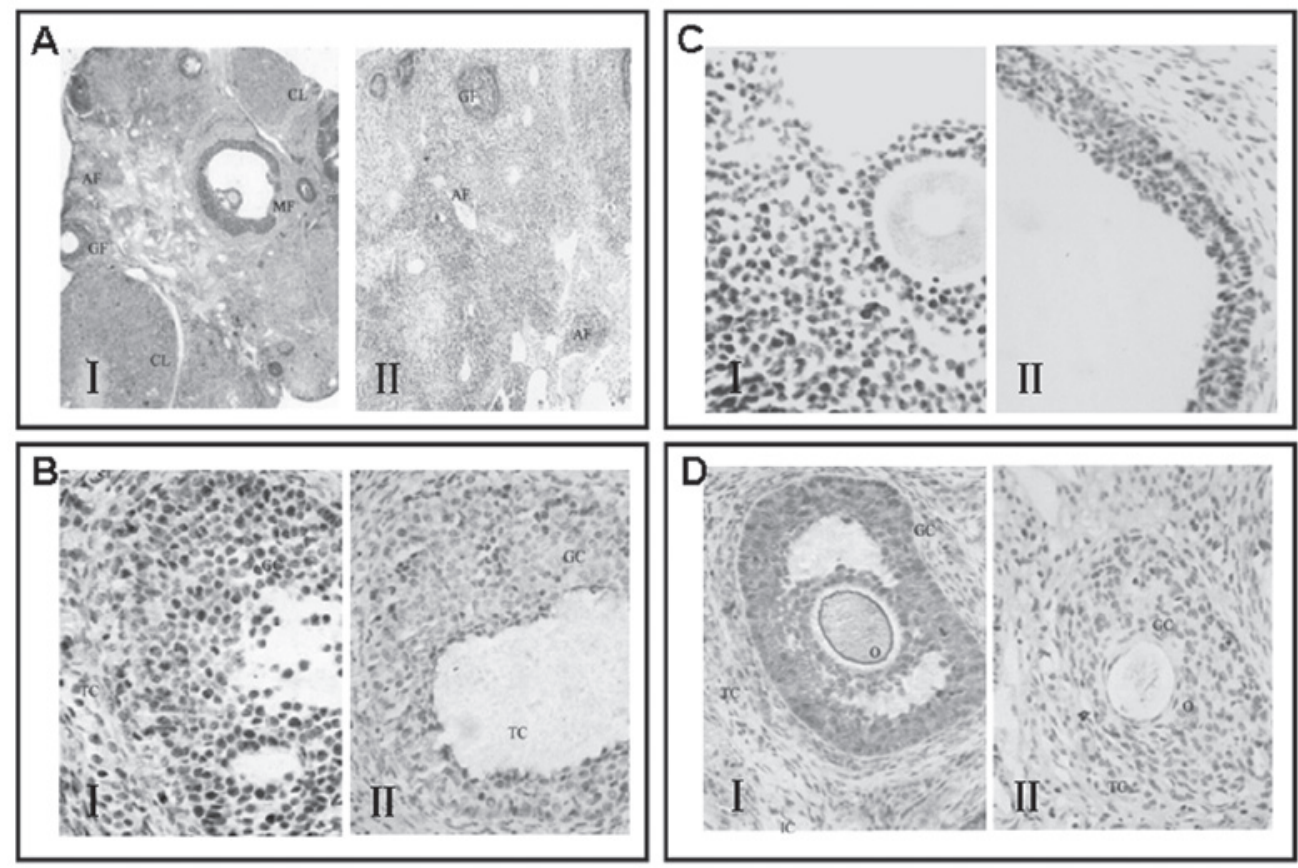

Figure 1. H\&E and immunohistochemical staining of ovary tissues in the two rat groups. (A) H\&E staining of ovary tissue of the control rats (I) and exercise training rats (II). Immunohistochemical staining of (B) ER, (C) PR and (D) estradiol in the ovary tissue of (I) control rats and (II) exercise training rats.

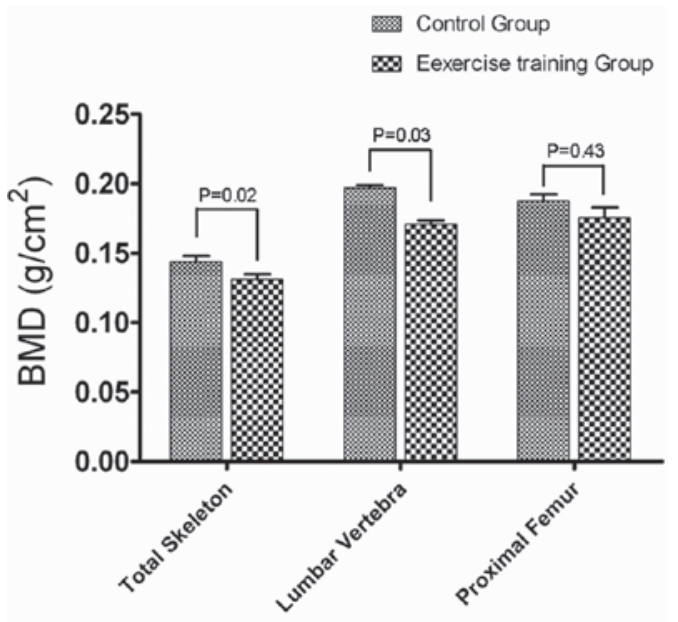

Figure 2. Bone mineral density (BMD) of the total skeleton, lumbar vertebra and proximal femur in the control group and exercise training group rats.

of DNA and its space of shift in gel, the standard curve was obtained with UTHSCSA ImageTool and Excel 2000 in each gel resulting in acquisition of the size (bp) of each fragment of DNA. According to the number of bp, genes were determined by searching in the corresponding database provided by Qbiogene, Inc. and MP Biomedicals, Inc. (http://www.qbiogene.com/displayfit/).

Quantitative RT-PCR. Rat ovaries were rapidly powdered in liquid nitrogen, and total-RNA was extracted using TRIzol (Invitrogen) according to the manufacturer's instructions. For quantitative real-time PCR, total-RNA was retrotranscribed using the RevertAid ${ }^{\mathrm{TM}}$ First Strand cDNA Synthesis kit (Fermentas) according to the manufacturer's instructions. PCR amplification and detection of the PCR amplified gene products were performed with the SYBR-Green PCR master mix (Tiangen). Real-time PCR was performed on Mastercycler ep realplex (Eppendorf). The reaction cycle consisted of hot start at $95^{\circ} \mathrm{C}$ for $10 \mathrm{~min}$, then 30 cycles of denaturation at $95^{\circ} \mathrm{C}$ for $30 \mathrm{sec}$, annealing at $60^{\circ} \mathrm{C}$ for $30 \mathrm{sec}$ and extension at $72^{\circ} \mathrm{C}$ for $30 \mathrm{sec}$. The primer sequences used for PCR are shown in Table I. Experiments were performed in triplicate in three independent experiments. Data were collected to the SYBR channel at the end of each extension step, and the final cycle was followed by a thermal melt step. Data collected for each sample were analyzed using the $2^{-\Delta \Delta \mathrm{CT}}$ method with GAPDH as an internal control (17).

\section{Results}

High intensity exercise training affects ovary morphology. There was a significant difference between the two rat groups in regards of body weight and the weights of the ovary and uterus (Table II). As for histological characteristics, the ovaries in the exercise training group rats showed a thinner cortex, more atretic follicles and less vessels and lymphatic vessels, as well as more corpus luteum atrophy. In contrast, in the control group, normal ovarian follicles in different stages were observed, together with more granular cells and fresh corpus luteum (Fig. 1A).

High intensity exercise training affects ovarian function. To evaluate the effect of high intensity exercise on the function of the ovaries, we measured the levels of circulating reproductive hormones such as estradiol, progesterone, $\mathrm{LH}$, and FSH and BMD. A significant decrease in plasma estradiol, progesterone, LH and FSH levels was noted (Table II). 
A

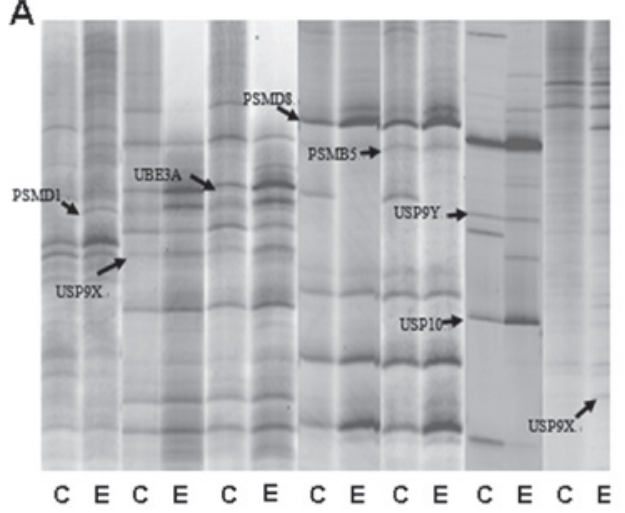

B

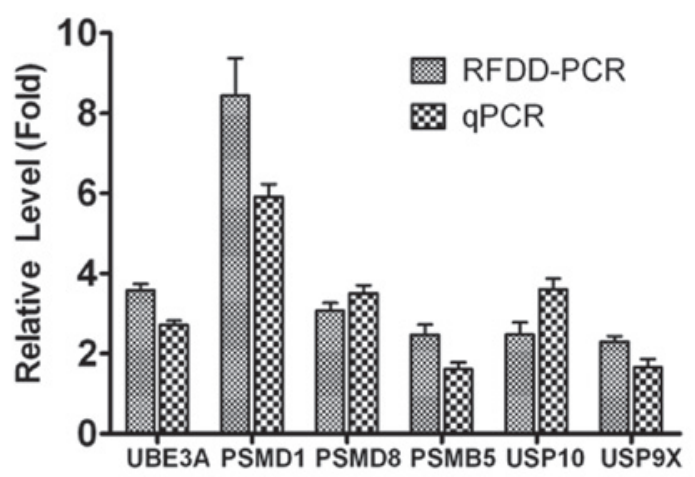

Figure 3. Differentially expressed genes in the control (C) and exercise training (E) rats by RFDD-PCR and real-time PCR. (A) Cy5-labeling 7\% urea polyacrylamide gels scanned with Typhoon 9200 Variable Mode Imager. Ubiquitin-proteasome system genes Psmb5, Psmd1, Psmd8, Usp9x, Usp10 and Ube3a were overexpressed in the exercise training rats. (B) Confirmation of RFDD-PCR data using real-time RT-PCR. Compared to the control rats, the expression levels of Psmb5, Psmd1, Psmd8, Usp9x, Usp10 and Ube3a were significantly upregulated in the exercise training rats as determined by real-time RT-PCR.

Immunohistochemical analysis demonstrated that the ovarian tissues were weakly stained for the estradiol, ER and PR antibodies in the rats from the high intensity exercise training group, but the staining was much stronger in the rats from the control group (Fig. 1B-D). As the major target tissue of ovary hormones, the bone tissue was also affected by high intensity exercise training. As revealed by DXA scanning, the BMD of the total skeleton and lumber vertebra in the exercise training group rats was significantly decreased $(\mathrm{P}<0.05)$ (Fig. 2).

High intensity exercise training affects gene expression of the ovaries. Differential gene expression profiles of the ovaries from the two rat groups were established using RFDD-PCR technique according to the company's operation manual. Here we separated and identified 3512 fragments. The sizes of these fragments were 50-1000 bp (Fig. 3A). To screen significantly altered gene expression, bioinformatics analysis was applied using the database of GenBank and ImageTool Software. There were 354 gene fragments that were found to be differentially expressed in the two rat groups. Among all of the differentially expressed gene fragments, genes of the ubiquitin-proteasome system including Psmb5, Psmd1, Psmd8, Usp9x, Usp10 and Ube $3 a$ were significantly upregulated in the high intensity excercise training group (Fig. 3A). To assess the validity of the RFDD-PCR data, real-time quantitative PCR was performed to compare the gene expression levels between the two rat groups. In all cases, RT-PCR data were consistent with the results of the RFDD-PCR data, suggesting that the dataset obtained from the RFDD-PCR data analysis accurately reflects gene expression differences between the exercise training and the control rats (Fig. 3B).

\section{Discussion}

In the present study, we investigated the effect of high intensity exercise training on the function and gene expression profile of ovaries. Our results showed that 8 weeks of high intensity exercise training significantly decreased the level of estradiol, progesterone, LH and FSH in plasma and BMD. RFDD-PCR and real-time PCR revealed that genes of the ubiquitin protea- some system such as Psmb5, Psmdl, Psmd8, Usp9x, Usp10 and $U b e 3 a$ were significantly upregulated in the high intensity exercise training rat ovaries. The results indicate that high intensity exercise training results in ovarian dysfunction by altering the expression of genes of the ubiquitin-proteasome system.

Regular aerobic exercise has been associated with many positive effects such as decreased heart rate and blood pressure and enhanced oxidative capacity (18). However, long-term high intensity exercise training results in a serious negative influence on numerous tissues or organs (19). Our data revealed a marked decrease in desquamated keratinocytes of the vagina and coefficient of both the ovaries and uterus in the experimental group, which indicated suppression of the reproductive system in the animal model. As the female gonad, ovaries secrete estrogen, progesterone, as well as produce ovum. The hormones of the ovary play an important role in the maintenance of a normal menstrual cycle and in the function of reproduction by regulating endometrial cyclical variation, while providing feedback to the hypothalamus and pituitary. We observed that expression of ER and PR decreased in granular cells and lutein cells in the exercise training rats, and a positive correlation was noted between the expression of ER and PR and estradiol level. This indicates that high intensity exercise training suppresses the expression of ER/PR and the secretion of estrogen in the ovary, thus influencing normal function of the ovary, including follicular development, ovulation, corpus luteum formation, gonadal hormone secretion and menstruation.

Through a complex process, long-term high intensity exercise training alters the morphology and function of the ovary which may relate to important gene expression alterations. In the present study, RFDD-PCR, a technique based on differential-display-PCR, combining fluorescence intensity of electrophoresis bands with expression abundance of mRNA, was used to establish the differential expression profile between high intensity exercise training rats and control rats. This method provides the opportunity to observe all alterations in the entire gene expression profile. Our data revealed that 354 gene fragments were differentially expressed between the two rat groups, indicating that high intensity exercise training 
affects the function of ovaries through a complex biological process involving a multi-gene family. Among the differentially expressed genes, the ubiquitin-proteasome system was the focus of our interest in analyzing the function of the differentially expressed genes. The ubiquitin-proteasome system plays a role in almost all processes involved in cell regulation, such as the cell cycle, signal transduction, proliferation and differentiation (5). Our data revealed 7 upregulated genes in the ubiquitin-proteasome system, including 3 related to subunits of the proteasome, 3 related to ubiquitin and 1 coding an ubiquitin-protein ligating enzyme UBE3A; this implies that there was abnormal activation of the ubiquitinproteasome system during the process of reproductive system dysfunction due to high intensity exercise training. Previous studies have shown that an activated ubiquitin-proteasome system affects oogenesis by targeted cyclin E $(20,21)$. In our study, we also found that ER and PR were downregulated in the ovaries in the exercise training group rats. Collectively, we hypothesized that high intensity exercise training upregulates the ubiquitin-proteasome system in ovaries to modulate an important signaling pathway, resulting in alterations in ovarian morphology and function.

In summary, we determined the differential gene expression profile between high intensity exercise training rats and control rats and identified that the ubiquitin proteasome system was activated in the high intensity exercise training rats. The activated ubiquitin-proteasome system may be involved in the alteration of ovarian morphology and function induced by long-term high intensity exercise training. Further investigation is required to confirm functionally relevant genes and signaling pathways involved in ovary dysfunction in vitro and in vivo.

\section{References}

1. Kent W: The effects of sprint interval training on aerobic fitness in untrained individuals: a systematic review. Br J Sports Med 45: A8, 2011

2. Manson JE and Lee IM: Exercise for women - how much pain for optimal gain? N Engl J Med 334: 1325-1327, 1996.

3. Warren MP and Perlroth NE: The effects of intense exercise on the female reproductive system. J Endocrinol 170: 3-11, 2001.

4. Safarinejad MR, Azma K and Kolahi AA: The effects of intensive, long-term treadmill running on reproductive hormones, hypothalamus-pituitary-testis axis, and semen quality: a randomized controlled study. J Endocrinol 200: 259-271, 2009.

5. Nalepa G, Rolfe M and Harper JW: Drug discovery in the ubiquitin-proteasome system. Nat Rev Drug Discov 5: 596-613, 2006
6. Bedford L, Lowe J, Dick LR, Mayer RJ and Brownell JE: Ubiquitin-like protein conjugation and the ubiquitin-proteasome system as drug targets. Nat Rev Drug Discov 10: 29-46, 2010.

7. Shen B, Zhang Z, Wang Y, et al: Differential expression of ubiquitin-conjugating enzyme E2r in the developing ovary and testis of penaeid shrimp Marsupenaeus japonicus. Mol Biol Rep 36: 1149-1157, 2009.

8. Huo LJ, Fan HY, Zhong ZS, Chen DY, Schatten H and Sun QY: Ubiquitin-proteasome pathway modulates mouse oocyte meiotic maturation and fertilization via regulation of MAPK cascade and cyclin B1 degradation. Mech Dev 121: 1275-1287, 2004.

9. Huo LJ, Fan HY, Liang CG, Yu LZ, Zhong ZS, Chen DY and Sun QY: Regulation of ubiquitin-proteasome pathway on pig oocyte meiotic maturation and fertilization. Biol Reprod 71: 853-862, 2004.

10. Suzumori N, Burns KH, Yan W and Matzuk MM: RFPL4 interacts with oocyte proteins of the ubiquitin-proteasome degradation pathway. Proc Natl Acad Sci USA 100: 550-555, 2003.

11. Chen GQ, Mou CY, Yang YQ, Wang S and Zhao ZW: Exercise training has beneficial anti-atrophy effects by inhibiting oxidative stress-induced MuRF1 upregulation in rats with diabetes. Life Sci 89: 44-49, 2011.

12. Sandri M, Podhorska-Okolow M, Geromel V, Rizzi C, Arslan P, Franceschi $\mathrm{C}$ and Carraro U: Exercise induces myonuclear ubiquitination and apoptosis in dystrophin-deficient muscle of mice. J Neuropathol Exp Neurol 56: 45-57, 1997.

13. Nedergaard A, Vissing K, Overgaard K, Kjaer M and Schjerling P: Expression patterns of atrogenic and ubiquitin proteasome component genes with exercise: effect of different loading patterns and repeated exercise bouts. J Appl Physiol 103: 1513-1522, 2007.

14. Sun B, Wang JH, Lv YY, Zhu SS, Yang J and Ma JZ: Proteomic adaptation to chronic high intensity swimming training in the rat heart. Comp Biochem Physiol Part D Genomics Proteomics 3: 108-117, 2008.

15. Deng S, Zhou H, Xiong R, et al: Over-expression of genes and proteins of ubiquitin specific peptidases (USPs) and proteasome subunits (PSs) in breast cancer tissue observed by the methods of RFDD-PCR and proteomics. Breast Cancer Res Treat 104: 21-30, 2007.

16. Zhou HY, Mei Y,Lu YG, et al: Application of restriction fragment differential display-polymerase chain reaction in study on differential expression profiles of human diseases. Zhonghua Yi Xue Yi Chuan Xue Za Zhi 22: 294-297, 2005.

17. Livak KJ and Schmittgen TD: Analysis of relative gene expression data using real-time quantitative PCR and the 2(-Delta Delta C(T)) method. Methods 25: 402-408, 2001.

18. Tjønna AE, Lee SJ, Rognmo Ø, et al: Aerobic interval training versus continuous moderate exercise as a treatment for the metabolic syndrome: a pilot study. Circulation 118: 346-354, 2008.

19. Zou J, Yuan J, Lv S and Tu J: Effects of exercise on behavior and peripheral blood lymphocyte apoptosis in a rat model of chronic fatigue syndrome. J Huazhong Univ Sci Technolog Med Sci 30: $258-264,2010$

20. Zhang PJ, Zhao J, Li HY, et al: CUE domain containing 2 regulates degradation of progesterone receptor by ubiquitinproteasome. EMBO J 26: 1831-1842, 2007.

21. Lonard DM and O'Malley BW: Emerging roles of the ubiquitin proteasome system in nuclear hormone receptor signaling. Prog Mol Biol Transl Sci 87: 117-135, 2009. 